\title{
Italian validation of the functional difficulties questionnaire (FDQ-9) and its correlation with major determinants of quality of life in adults with hypermobile Ehlers-Danlos syndrome/hypermobility spectrum disorder
}

\begin{tabular}{|c|c|}
\hline Journal: & American Journal of Medical Genetics Part B: Neuropsychiatric Genetics \\
\hline Manuscript ID & NPG-18-0060.R1 \\
\hline Wiley - Manuscript type: & Research Article \\
\hline Date Submitted by the Author: & $\mathrm{n} / \mathrm{a}$ \\
\hline Complete List of Authors: & $\begin{array}{l}\text { Morlino, Silvia; Division/Laboratory of Medical Genetics, Department of } \\
\text { Molecular Medicine, Sapienza University, San Camillo-Forlanini Hospital, } \\
\text { Rome, Italy } \\
\text { Dordoni, Chiara; School of Medicine, University of Brescia, Division of } \\
\text { Biology and Genetics, Department of Molecular and Translational Medicine } \\
\text { Sperduti, Isabella; IRCCS-San Gallicano Dermatologic Institute } \\
\text { Clark, Carol; Bournemouth University, Department of Human Sciences and } \\
\text { Public Health } \\
\text { Piedimonte, Caterina; Sapienza University of Rome, Policlinico Umberto I } \\
\text { Hospital, Division of Child Neurology and Psychiatry, Department of Human } \\
\text { Neurosciences } \\
\text { Fontana, Andrea; IRCCS-Casa Sollievo della Sofferenza Hospital, Unit of } \\
\text { Biostatistics } \\
\text { Colombi, Marina; School of Medicine, University of Brescia, Division of } \\
\text { Biology and Genetics, Department of Molecular and Translational Medicine } \\
\text { Grammatico, Paola; Medical Genetics, Experimental Medicine } \\
\text { Copetti, Massimiliano; IRCCS-Casa Sollievo della Sofferenza, Unit of } \\
\text { Biostatistics } \\
\text { Castori, Marco; IRCCS-Casa Sollievo della Sofferenza Hospital, Medical } \\
\text { Genetics }\end{array}$ \\
\hline Keywords: & $\begin{array}{l}\text { coordination, Ehlers-Danlos syndrome, joint hypermobility, quality of life, } \\
\text { pain }\end{array}$ \\
\hline
\end{tabular}

\section{SCHOLARONE




\title{
Italian validation of the functional difficulties questionnaire (FDQ-9) and its correlation with major determinants of quality of life in adults with hypermobile Ehlers-Danlos syndrome/hypermobility spectrum disorder
}

\author{
Silvia Morlino, ${ }^{1,2}$ Chiara Dordoni, ${ }^{3}$ Isabella Sperduti, ${ }^{4}$ Carol J. Clark, ${ }^{5}$ Caterina Piedimonte, ${ }^{2,6}$ Andrea \\ Fontana, ${ }^{7}$ Marina Colombi, ${ }^{3}$ Paola Grammatico, ${ }^{1}$ Massimiliano Copetti, ${ }^{7}$ and Marco Castori. ${ }^{8}$ \\ 1: Laboratory of Medical Genetics, Department of Molecular Medicine, Sapienza University of Rome, \\ San Camillo-Forlanini Hospital, Rome, Italy. 2: "Vaclav Vojta" Rehabiliation Center, Rome, Italy. 3: \\ Division of Biology and Genetics, Department of Molecular and Translational Medicine, School of \\ Medicine, University of Brescia, Brescia, Italy. 4: Biostatistics, IRCCS-San Gallicano Dermatologic \\ Institute, Rome, Italy. 5: Department of Human Sciences and Public Health, Bournemouth University, \\ Bournemouth, United Kingdom. 6: Department of Experimental Medicine, Sapienza University, Rome, \\ Italy. 7: Unit of Biostatistics, IRCCS-Casa Sollievo della Sofferenza, San Giovanni Rotondo (FG), Italy. 8: \\ Division of Medical Genetics, IRCCS-Casa Sollievo della Sofferenza, San Giovanni Rotondo (FG), Italy.
}

Short title: FDQ9 and its clinical relevance in adults with hEDS/HSD.

Funding: None.

Conflict of Interest: All authors declare no conflict of interest concerning this work.

\author{
Correspondence to: Marco Castori, MD, PhD \\ Chief, Division of Medical Genetics \\ Foundation IRCCS-Casa Sollievo della Sofferenza \\ Poliambulatorio "Papa Giovanni Paolo II" \\ Viale Padre Pio, 7 \\ 71013 San Giovanni Rotondo (FG) \\ Italy \\ Phone: +39-0882416288 \\ Fax: +39-0882411616 \\ Email: m.castori@operapadrepio.it
}




\begin{abstract}
The 2017 Nosology defines the new criteria for hypermobile Ehlers-Danlos syndrome (hEDS), which is now considered one end of a continuous spectrum encompassing isolated, non-syndromic joint hypermobility (JH) and hypermobility spectrum disorders (HSD). Preliminary data indicate a link between $\mathrm{JH}$ and neurodevelopmental disorders and, in particular, developmental coordination disorder (DCD) in children. Assessing DCD in adults is difficult and the recently described functional difficulties questionnaire 9 (FDQ-9) is one of the few available tools. The aims of this study are to (i) validate FDQ-9 written in Italian and present normal values in 230 Italian controls; and (ii) explore the relationship of FDQ-9 with the brief pain inventory, composite autonomic symptom score 31, multidimensional fatigue inventory, attention deficit/hyperactivity disorder self-report version 1.1, and the SF-36 for quality of life in 105 Italian adults with hEDS/HSD. Validation of the FDQ-9 in Italian was carried out by translation, cross-cultural adaptation and test/retest reliability analysis. A casecontrol study was performed comparing the FDQ-9 outcome between 105 patients and 105 sex-and age-matched controls. Fifty-nine percent of the patients resulted positive compared to the $3.8 \%$ of controls ( $p$-value $<0.00001$ ). In patients, FDQ-9 positive result associated with positive attention deficit/hyperactivity disorder self-report version $1.1(\mathrm{OR}=4.04)$. Multivariate regression analysis comparing FDQ-9 with the other questionnaires demonstrated a strong association between positive FDQ-9 and the number of painful joints. Our preliminary data open wider management and therapeutic perspectives for coordination difficulties in hypermobile individuals.
\end{abstract}

Keywords: coordination, dysautonomia, Ehlers-Danlos syndrome, joint hypermobility, quality of life, pain. 


\section{Introduction}

Hypermobile Ehlers-Danlos syndrome (hEDS) is probably the most common heritable connective tissue disorder and remains without known molecular bases (Tinkle et al., 2017). The 2017 international classification of Ehlers-Danlos syndromes (EDS) and related disorders identifies stricter criteria for hEDS that is defined by the presence of generalized joint hypermobility (GJH) (criterion 1); two or more items among systemic involvement, positive family history and musculoskeletal involvement (criterion 2); and the exclusion of other hereditary and acquired connective tissue disorders (criterion 3) (Malfait et al., 2017). hEDS is also considered at one end of a continuous phenotypic spectrum which originates from isolated, non-syndromic joint hypermobility (JH) and passes through the recently defined hypermobility spectrum disorders (HSDs) (Castori et al., 2017). HSDs are terms used to defined all symptomatic individuals with $\mathrm{JH}$ who do not meet the new diagnostic criteria of hEDS (Castori \& Hakim, 2017).

One of the aims of the 2017 international classification was to solve the nosologic conundrum of the hEDS and the "old" joint hypermobility syndrome (JHS). Previous literature identified hEDS with the Villefranche criteria (Beighton, De Paepe, Steinmann, Tsipouras, \& Wenstrup, 1998) and the JHS by the Brighton criteria (Grahame, Bird, \& Child, 2000), a fact suggesting that the two disorders are distinct. One decade afterwards, a community of experts declared that the two disorders are indistinguishable on clinical grounds and, therefore, that they should be considered a single disorder (Tinkle et al., 2009). Now, hEDS is recognized by novel criteria, and the JHS is removed and incorporated within the HSDs, which do not overlap hEDS.

In hEDS/HSD, quality of life (QoL) is frequently affected because of chronic pain and fatigue (Castori et al., 2012; Castori et al., 2013; Pacey, Tofts, Adams, Munns \& Nicholson, 2015), and partly influenced by autonomic dysfunction (De Wandele et al., 2016). Co-morbidities are strong QoL determinants in hEDS/HSD, but the nature of their link with JH remains obscure. Fragmented data indicate that the association between $\mathrm{JH}$ and neurodevelopmental disorders is a potential field of interest. Daily practice on children with hEDS/HSD suggests a high rate of developmental motor and coordination disorders (Ghibellini, Brancati \& Castori, 2015). In 2005, Adib, Davies, Grahame, Woo \& Murray reported clumsiness and symptoms of poor coordination in 125 children with hEDS/HSD. In two studies, Kirby and colleagues suggested functional similarities between children with GJH or hEDS/HSD and those with developmental coordination disorder (DCD) (Kirby, Davies \& Bryant, 2005; Kirby \& Davies, 2007). Accordingly, GJH is more frequent in children with DCD (Jelsma, Geuze, Klerks, Niemeijer \& Smits-Engelsman, 2013; Celletti et al., 2015) as well as in those with the commonly comorbid attention deficit and hyperactivity disorder (ADHD), compared to controls (Koldas Dogan, 
Taner \& Evcik,2011; Sinibaldi, Ursini \& Castori, 2015). Nevertheless, DCD and ADHD are well-defined diagnoses only in the developmental age.

The functional difficulties questionnaire 9 (FDQ-9) and ADHD self-report version 1.1 (ASRS-V1.1) are among the few tools able to explore DCD- and ADHD-related symptoms in adults (Kessler et al., 2005; Clark, Thomas, Khattab \& Carr, 2013). The FDQ-9 was first presented in 2013 as a reliable tool for screening adults for a possible diagnosis of DCD. In the original paper, the general psychometric properties of FDQ-9 were investigated in 167 subjects from the general population and 90 patients who attended a hypermobility clinic (Clark, Thomas, Khattab \& Carr, 2013). In a comparable sample of $90 \mathrm{JHS}$ adults, the same group demonstrated a $56 \%$ prevalence of positive FDQ-9 screening, with a 5.5 odds ratio compared to 113 healthy volunteers (Clark, Khattab \& Carr, 2014). In a further work, the significance of FDQ-9 in relationship with the SMART wobbleboard was explored in healthy adults (Clark, Clark, Dorey \& Williams, 2016). Nevertheless, studies exploring the relevance of this test in a clinical setting are still lacking.

This work was aimed to (i) validate FDQ-9 in Italian and present its pattern in 230 Italian controls, and (ii) explore, for the first time, the relationships of FDQ-9 with the brief pain inventory (BPI) for pain, composite autonomic symptom score 31 (COMPASS-31) for autonomic symptoms, multidimensional fatigue inventory 20 (MFI-20) for fatigue, ASRS-V1.1 for ADHD features, and the Short Form-36 (SF-36) for QoL in 105 Italian adults with hEDS/HSD. Our study adds information for deciphering the complexities of body-mind connections in $\mathrm{JH}$ and related disorders.

\section{Patients and Methods}

\section{Patients' and controls' selection}

Patients were enrolled from February 2016 to February 2017 in two expert Italian centers for JH and related disorders, i.e. the Medical Genetics Clinic of the San Camillo-Forlanini Hospital (Rome) and the Center for Diagnosis and Management of EDS and Hereditary Connective Tissue Disorders at the Spedali Civili University Hospital (Brescia). All patients underwent a systematic evaluation as previously described (Castori et al., 2014; Castori \& Colombi, 2015). As data for this study were obtained before the publication of the 2017 International Classification, patients were originally selected according to the Villefranche and Brighton criteria (Beighton, De Paepe, Steinmann, Tsipouras \& Wenstrup, 1998; Grahame, Bird \& Child, 2000). Retrospectively, clinical data of selected patients were compared to the hEDS criteria of the 2017 International Classification and the alternative diagnoses of hEDS and HSD were annotated. In case of a suspected overlap with other acquired or hereditary connective tissue disorders, differential diagnosis extended to autoimmune rheumatologic screening, heart ultrasound, bone densitometry and other selected supplementary 
ascertainments (i.e. ophthalmologic survey, brain and spine MRI) . When necessary, other sets of criteria, such as the revised Ghent criteria for Marfan syndrome (Loeys et al., 2010), were applied together with the use of appropriate molecular studies. No patients had evidence of intellectual disability or autism spectrum disorder.

Italian controls were enrolled from colleagues and friends of the authors, as well as from the healthy individuals and their relatives attending prenatal screening at the San Camillo-Forlanini Hospital. Among these controls, three were bilingual native Italian speakers (see below). All clinical and controls gave their consent to the study, which is in accordance to the revised version of the Helsinki Declaration. This study was approved by the Local Ethics Committee (protocol no. 250/CE Lazio 1).

\section{Translation and cross-cultural adaptation of FDQ-9}

The FDQ-9 is a 9-items self-assessment instrument to indicate functional difficulties associated with DCD/dyspraxia, previously validated in English patients by Clark and colleagues (Clark, Thomas, Khattab \& Carr, 2013). For each question, "very good" answers were calculated as 1 point, "good" as 2 points, "poor" as 3 points, and "very poor" as 4 points. According to the original description of FDQ-9 (Clark, Thomas, Khattab \& Carr, 2013), the cut-off above which functional difficulties impacting daily functional activities was fixed at 21.5 , while a result ranging from 19 to 21 was considered borderline for difficulties impacting daily function. A score of 18 or below was considered negative for functional difficulties.

Three bilingual native Italian speakers independently translated the FDQ-9 questionnaire into Italian (forward translation phase). Subsequently, a consensus was reached on a first preliminary Italian version based on the three translations. Next, two bilingual native English speakers retranslated the Italian version into English (back translation phase). Any inconsistency was resolved. Reproducibility, or test/retest reliability, was assessed using the questionnaires of 45 individuals (20 hEDS/HSD and 25 controls) who completed the questionnaire and a second copy in a period of eight weeks after the first administration.

\section{Reference values of FDQ-9 in Italian controls}

Once translated and cross-culturally adapted, the Italian version of the FDQ-9 was administered to 230 Italian controls. Results from these 230 healthy individuals were used to trace the reference values of the FDQ-9 in the Italian general population.

Psychometric evaluation of hEDS/HSD adults 
One-hundred and five, directly evaluated hEDS/HSD adults underwent a series of psychometric tests after medical examination and diagnosis review during clinical follow-up at their reference medical center. Used tests included: FDQ-9 (see above for details), ASRS-V1.1,BPI, COMPASS-31, MFI20, and SF-36. From the 230 Italian controls, a sample of 105 healthy individuals was extracted, matched for age and sex with the hEDS/HSD patients' group. This subsample was used as controls for the interpretation of the FDQ-9 results in hEDS/HSD patients.

\section{ASRS-V1.1}

The attentive-executive domain was evaluated in the patients' sample by the ASRS-V1.1, which was originally developed for adults screening by the WHO together with revision of the WHO Composite International Diagnostic Interview (Kessler et al., 2005; Kessler et al., 2007). The ASRSV1.1 is a symptom checklist for the DSM-IV-TR criteria of ADHD (APA, 2000). The six questions of the part A of ASRS-V1.1 were found to be the most predictive for ADHD and were considered the basis for the ADHD screening in adults. ASRS-V1.1 was given to patients in its Italian version (freely downloadable at: https://www.aifa.it/documenti/ASRSV1.1.pdf).The sum of the six answers corresponds to how the adult patient has felt and acted in the last 6 months. The cut-off for suspecting ADHD in adults is currently 4 or more positive answers, calculated on the basis of frequency for each item. The results emerging from this evaluation may suggest the need for a more in-depth clinical analysis in order to confirm or discard the diagnosis, which remains difficult in adults. However, ASRS-V1.1 was calibrated to DSM-IV-TR criteria, which are narrower than the recently developed DSM-5 criteria (APA, 2013). The present work started before the publication of the DSM-5 updated version of this questionnaire, in which, however, the six questions of part A remain unchanged (Ustun et al., 2017). ASRS-V1.1 was never used in hEDS/HSD.

$B P I$

The Italian version of BPI was used to explore pain features in hEDS/HDS adults (Caraceni et al., 1996). BPI is a self-administered multidimensional questionnaire for the evaluation of pain and analyzes four domains including history, location, intensity and interference with daily functions (Cleeland \& Ryan, 1994). BPI can be used in adults and, consists of 13 items exploring time course of pain, pain extension, pain intensity, pain therapies, pain interference with motor and relational activities, and with sleeping and mood. For the present study, replies to questions no 3 to 6,8 and 9 were used as continuous variables. Concerning question no. 2 (homunculus for painful sites pointed by the patient with a pen), each painful point counted 1 and the sum was used as a continuous variable ("bodily pain"). In case of painful points clearly corresponding to joints a second continuous variable was extracted, specific for "joint pain". No previous study on EDS has applied this test. 


\section{MFI-20}

MFI-20 was used for assessing fatigue in hEDS/HSD patients by using its Italian version (JereczekFossa et al., 2007). MFI-20 is a 20-item self-report scale that measures fatigue symptoms in adults. Items are grouped into five areas (general fatigue, physical fatigue, mental fatigue, reduced motivation and reduced activity) each composed of four items (Smets, Garssen, Bonke \& De Haes, 1995). All items require an evaluation on a 5-point scale, ranging from an answer in accordance with the question "yes, it's true" (= 1 point) to an answer of total disagreement "no, it's not true" (= 5 points). For scoring, it is first necessary that items $2,5,9,10,13,14,16,17,18$ and 19 are overturned ( 1 point $\rightarrow 5$ points, 2 points $\rightarrow 4$ points, 3 points $\rightarrow 3$ points , 4 points $\rightarrow 2$ points, 5 points $\rightarrow 1$ point). Items compose the five areas as follows: items 1, 5, 12 and 16 refer to "general fatigue" (MFI20-GF); items 2, 8, 14 and 20 refer to "physical fatigue"(MFI20-PF); items 3, 6, 10 and 17 refer to "reduction of activities"(MFI20-RA); items 4, 9, 15 and 18 refer to "reduction of motivation"(MFI20RM); and items 7, 11, 13 and 19 refer to "mental fatigue"(MFI20-MF). Accordingly, all areas have a total subscale score ranging from 4 to 20 . A higher score in each item and subscale is equivalent to a greater presence of related symptoms. The "general fatigue" subscale is considered the best indicator to assess fatigue with a single score (Smets, Garssen, Bonke \& De Haes, 1995). MFI-20 was previously used in hEDS by our group in a single study (Celletti, Castori, La Torre \& Camerota, 2013).

\section{COMPASS-31}

COMPASS-31 is a self-assessment instrument to evaluate dysautonomic symptoms based on 31 items grouped in six autonomic domains, including 4 items for orthostatic intolerance (COMPASS31OI), 3 items for vasomotor (COMPASS31-VM), 4 items for secretomotor (COMPASS31-SM), 12 items for gastrointestinal (COMPASS31-GI),3 items for bladder (COMPASS31-B), and 5 items for pupillomotor (COMPASS31-PM) functions (Sletten, Suarez, Low, Mandrekar \& Singer, 2012). An Italian version of COMPASS-31 was previously validated and translated (Pierangeli et al., 2015). For scoring simple questions (e.g. presence vs absence), negative and positive answers were calculated as 0 and 1 points, respectively. Frequency of symptoms was scored as 0 points for rarely or never reported, 1 point for occasionally or sometimes reported, 2 points for frequently reported or reported "a lot of the time", and 3 points for almost always or constantly reported. Severity of symptoms was calculated as 1 point for mild degree, 2 points for moderate degree, and 3 points for severe degree. For the time course of a symptom, 0 points were attributed for responses such as "gotten somewhat better", "gotten much better", "completely gone", and "I have not had any of these symptoms"; 1 point for "stayed about the same"; 2 points for "gotten somewhat worse"; and 3 
points for "gotten much worse". Every domain is considered as single score derived by the sum of related items. No previous study on EDS applied COMPASS-31.

SF-36

Health status and particularly QoL were assessed by administering the SF-36 questionnaire to the patients' sample. SF-36 has an Italian version (Apolone \& Mosconi, 1998), which was previously used in EDS adults by our group and others (Castori, Camerota, Celletti, Grammatico \& Padua, 2010; Rombaut, Malfait, Cools, De Paepe \& Calders, 2010; Celletti, Castori, La Torre \& Camerota, 2013). SF36 is a useful self-report 36-item questionnaire consisting of eight subscales that describe physical activity (PA), physical role limitations (PRL), physical pain (PP), general health (GH), vitality (V), social activity (SA), emotional role limitations (ERL), and mental health $(\mathrm{MH})$ over the past four weeks. Every scale ranges from 0 to 100, with 0 indicating the negative lowest score and 100 indicating the positive highest score. These eight scales are then grouped in three areas, named physical health $(\mathrm{PH}=\mathrm{PA}+\mathrm{PRL}+\mathrm{PP})$, general health $(\mathrm{GH}+\mathrm{V})$ and psychological-emotional health $(\mathrm{PEH}=\mathrm{SA}+\mathrm{ERL}+\mathrm{MH})$. These scales were calculated using the algorithm freely available online and fully applicable to the Italian version of SF-36 (freely downloadable at: http://crc.marionegri.it/sf36/sf36v1ita.htm).

\section{Statistical methods}

Demographic and clinical characteristics were reported as medians with range and as frequencies and percentages for continuous and categorical variables, respectively. Test/retest reliability was measured by the intraclass correlation coefficient (ICC) and by the Pearson correlation coefficient(r). Group comparisons (case/control, positive/negative FDQ-9) were performed using Chi-square test and the non-parametric Mann-Whitney $U$ test for categorical (e.g. positive/negative ASRS-V1.1) and continuous (i.e. scores at the various items, domains and areas of BPI, COMPASS-31, MFI-20, and SF36) variables, respectively. Multivariate logistic regression analysis was performed to assess the association between FDQ-9 (positive/negative) with BPI, COMPASS-31, MFI-20, and SF-36. Risks were reported as odds ratios (ORs). Two-sided P-values $<0.05$ were considered statistically significant. All the analyses were performed using SAS Release 9.4 (SAS Institute, Cary, NC) and SPSS software (SPSS version 21.0, SPSS Inc., Chicago, Illinois, USA).

\section{Results}

\section{Demographic data}

One hundred and five adults with hEDS (Villefranche nosology) and/or JHS (Brighton criteria) were selected. Ninety-six were females and 9 males (female/male ratio: 10.6/1) with an age ranging from 18 to 62 years (mean: 36.9 years, standard deviation: 12.08). Their diagnosis was revised

John Wiley \& Sons, Inc. 
retrospectively, according to the 2017 International Classification of EDS and related disorders, and, hence, translated into the novel nosology of hEDS and HSD (Fig. 1). The distribution of the Beighton score in the patients' sample is reported in Fig. 2. The 230 controls from the general population included 138 females and 92 males with an age range of 19 to 95 years (mean 38.43 years; standard deviation: 12.20). Compared to the 105 patients, the sex- and age-matched 105 controls were 96 females and 9 males ( $p$-value: 1.00) with an age range from 19 to 62 years (mean: 37.2 years, standard deviation: $12.31 ; p$-value: 0.87$)$.

Validation of FDQ-9 in Italian

The translation in Italian of the FDQ-9 questionnaire is reported in Table I. It reported an high degree of test/retest reliability both in the control group $(n=20)$ as measured by the ICC $=0.965$ and by the Pearson correlation coefficient $r=0.934(p<0.0001)$ and in the patients group $(n=25)$ as measured by the ICC $=0.966$ and by the Pearson correlation coefficient $r=0.935(p<0.0001)$. Normal standards for FDQ-9 in Italian controls and results of FDQ-9 in hEDS/HSD adults and controls

After translation and validation in Italian, the results of FDQ-9 in the 230 Italian controls were represented in a bar graph (Fig.3). In this non-clinical sample of 230 individuals, 6 (2.6\%) resulted positive with a score above the cut-off ( $>21)$.

Comparison of the FDQ-9 results between the 105 adults with hEDS/HSD and 105 matched controls is summarized in Fig. 4. The patterns of replies were significantly different between the groups for all the 9 items, with an excess of "positive" replies ("poor" and "very poor") in the patients' cohort. An overt excess of individuals with a score above the cut-off (>21) was registered among patients $(62 / 105,59.0 \%)$ compared to controls $\left(4 / 105,3.8 \% ; \chi^{2}=74.33 ; p\right.$-value $=<0.00001$; Odds ratio $=36.41)$. We further analyzed the patients' and controls' samples by distinguishing between males and females, and by separating the samples in three categories as subsequently proposed by Clark, Clark, Dorey, \& Williams, 2016 (i.e. $\leq 18$ = no functional difficulties; 19-21 = at risk of having functional difficulties; $>21$ = with one or more functional difficulties). Results are summarized in Fig. 5.

Relationships of the FDQ-9 with the other questionnaires in hEDS/HSD adults

In the 105 adults with hEDS/HSD, the results of the FDQ-9 were compared to those of the other questionnaires (ASRS-V1.1, BPI, COMPASS-31, MFI-20 and SF-36). The comparison between the outcome to FDQ-9 (positive $>21$; negative $\leq 21$ ) and ASRS-V1.1 (positive $\geq 4$; negative $<4$ ) is reported in Fig. 6. Of the 105 hEDS/HSD, 55 (52.4\%) resulted positive for the ASRS-V1.1 screening for ADHD. Positivity to the FDQ-9 questionnaire was significantly associated with a positive result to ASRS-V1.1 in hEDS/HSD adults with a $p$-value of 0.0007 and an OR of 4.04 (95\%IC $=3.26-4.82)$. Results of the 
univariate analysis comparing the binary outcome to the FDQ-9 questionnaire (positive >21; negative 521) and the various items, domains and areas of the BPI, MFI-20, COMPASS-31 and SF-36 questionnaire (continuous variables) are reported in Table II. In summary, positive associations were identified for multiple items/domains/areas of BPI, COMPASS-31 and SF-36, while no significant finding occurred for MFI-20 (except for mental fatigue; MFI20-MF).The strongest associations ( $p$ value $<0.002$ ) were the number of painful joints $(\mathrm{BPI})$, pain interference with work and sleep (BPI), symptoms of orthostatic intolerance and functional gastrointestinal symptoms (COMPASS-31), and effects on the psycho-emotional health (SF-36). Multivariate logistic regression analysis nullified all significant associations of the univariate analysis, except for the number of painful joints at the BPI $(\mathrm{OR}=1.25,95 \% \mathrm{Cl}=1.06-1.47, p$-value $=0.008)$.

\section{Discussion}

This work includes the validation of the FDQ-9 questionnaire in a second language and, for the first time, explores its impact on selected determinants of QoL. In particular, we realized a highly confident Italian translation of the FDQ-9 (Table I) which showed significantly high scores at the test/retest analysis. In addition, we applied FDQ-9 in a large control sample in order to present some normative values in the Italian population, which may be useful for future studies in different clinically relevant disorders potentially affecting coordination and manifesting related functional difficulties. According to previous studies on FDQ-9 (Clark, Thomas, Khattab \& Carr, 2013; Clark, Khattab \& Carr, 2014; Clark, Clark, Dorey, \& Williams, 2016), we investigated the impact of this screening tool in patients with symptomatic $\mathrm{JH}$, who are known to suffer from clinical manifestations potentially related to coordination difficulties, especially in their developmental years (Kirby, Davies \& Bryant 2005; Kirdy \& Davies, 2007; Piedimonte et al., 2018). Such data provides a foundation of evidence around $\mathrm{JH}$ and its potential clinical reverberations.

The clinical boundaries of JH are broadening and the current nosology of EDS and related disorders extends to address a better classification of all patients with JH. Accordingly, individuals are now distinguished in subjects with (i) isolated, non-syndromic $\mathrm{JH}$, (ii) well-defined syndromes with $\mathrm{JH}$ (also comprising all Mendelian disorders with JH as well as the newly defined hEDS), and (iii) HSDs (Castori et al. 2017; Castori \& Hakim, 2017). At the same time, the existence of a continuous spectrum is accepted ranging from isolated, non-syndromic JH, to the various HSDs, to hEDS. This spectrum is the background family of phenotypes, in which patients with various forms of JH (symptomatic and asymptomatic, isolated and combined with other features), who are not associated with a specific genetic defect, are grouped. Isolated, non-syndromic JH and HSD are, in turn, subclassified according to the type of JH. In fact, patterns of JH include generalized JH, localized 
$\mathrm{JH}$, peripheral $\mathrm{JH}$ and historical $\mathrm{JH}$ on the basis of the Beighton score, 5-point questionnaire and presence/absence of JH in joints outside the Beighton score (Castori et al., 2017; Castori \& Hakim, 2017). This study focused on "symptomatic" patients belonging to the above described spectrum (i.e. patients affected by HSDs and hEDS).

Within such a heterogeneous group of patients, who, in the past, were alternatively diagnosed according the "old" Brighton and Villefranche criteria, increasing evidence is drawing an unexpected association with a series of neuropsychiatric and neurodevelopmental attributes (Sinibaldi, Ursini \& Castori, 2015; Ghibellini, Brancati \& Castori, 2015; Baeza-Velasco, Sinibaldi \& Castori, 2018). At the moment, the strongest association is between JH (usually, GJH) and anxiety disorders in adults (Bulbena et al., 2017). This prompted the Spanish group to propose the existence of a "neuroconnective phenotype" to define the heterogeneous pattern of psychosomatic reverberations of JH in adults (Bulbena, Pailhez, Bulbena-Cabré, Mallorquí-Bagué \& Baeza-Velasco, 2015).

Furthermore, $\mathrm{JH}$ is usually a congenital trait especially in individuals with generalized and/or bilateral forms of JH. This assumption has opened speculations on a possible "neurodevelopmental effect" of this trait in the developing child. Fragmented data suggest some overlap between the neuromuscular complaints of children with JH and those with DCD (Adib, Davies, Grahame, Woo \& Murray, 2005; Kirby, Davies \& Bryant, 2005; Kirby \& Davies, 2007). We recently contributed to this issue by comparing 23 children affected by hEDS/HSD, classical EDS and Loeys-Dietz syndrome with a control group of children primarily ascertained for DCD (Piedimonte et al., 2018). In the "syndrome" group we found $D C D, A D H D$ and learning disabilities in $30 \%, 13 \%$ and $22 \%$ of the cases, respectively. Conversely, in the DCD group, none had full characteristics of hEDS/HSD or other Mendelian disorders with $\mathrm{JH}$; a fact which suggests a low rate of "syndromic diagnoses" in children primarily ascertained for DCD. The pattern of neuropsychiatric and neurodevelopmental attributes of children with syndromic DCD (first group) and whose with non-syndromic DCD (second group) significantly differed for the severity and range of somatic complaints and behavioral traits (Piedimonte et al., 2018).

In the present work, we formalized the known association between hEDS/HSD, DCD and ADHD in adults. Although the diagnosis of DCD and ADHD are difficult in the post-developmental age, the two applied screening tools, i.e. FDQ-9 and ASRS-V1.1, are considered reliable for a clinical suspicion of these conditions in adults. Accordingly, $59 \%$ and $52 \%$ of the sample resulted positive to FDQ -9 and ASRS-V1.1, respectively. This rate is likely to be an overestimation as the data relates to self-reported complaints. This is partly supported by the lower rate of proved diagnoses of DCD and ADHD in our previous "pediatric" study (Piedimonte et al., 2018). Nevertheless, our data demonstrate a high rate of complaints related to impaired coordination and attention in adults with hEDS/HSD. Interestingly, 
the rate of 59\% of "positive" FDQ-9 (i.e. >21) in the patients' sample is comparable to the $56 \%$ in a previous work on 90 women with JHS by Clark, Khattab \& Carr (2014). Our findings also mirrored what happens in the general pediatric population in which ADHD and DCD are common comorbidities. This is reflected by the results of the FDQ-9 and ASRS-V1.1, which are significantly associated in our clinical sample. The strong association between positive ASRS-V1.1 for ADHD and hEDS/HSD in our study confirms what has previously been explored by a Swedish retrospective nationwide study which showed that EDS seems to predispose to ADHD with a 5.6 relative risk (Cederlöf et al., 2016). These findings may be interpreted in line with the well-known impairment of proprioception and fear of falling in adults with JH or hEDS (Rombaut et al., 2011; Scheper et al., 2017).

Multivariate logistic regression analysis showed a strong association between positive FDQ-9 and the number of painful joints in adults with hEDS/HSD. Interpretation of this finding is not clear-cut. However, some hypothesis can be put forward. First, a "clumsy" individual is more prone to softtissue injuries, falls and joint traumas; a mechanism that might explain a high rate of post-traumatic joint pain in adults with $\mathrm{JH}$ and features of $\mathrm{DCD}$. Available data also suggest a link between clumsiness and impaired proprioception in individuals with $\mathrm{JH}$. Therefore, it is possible that subjects who are born with $\mathrm{JH}$ also present an impaired proprioception during their developmental age. It is presumed by the recent identification of significant impairment of postural controls in children with EDS (Lisi et al., 2017). This might facilitate the instauration of atypical motor schemes which predispose a lax joint to microtraumas and, hence, to the development of recurrent and chronic joint pain in children and adults. Therefore, our findings reinforce the link between the severity of functional difficulties and the rate of chronic widespread pain in hEDS/HSD, as previously hypothesized in JHS adults by Clark, Khattab \& Carr (2014). In this perspective, in the hypermobile child, the presence of DCD might be predictive for the severity of joint pain (in terms of number of painful joints) in adulthood. This scenario is tempting for a possible preventive effect of proprioceptive improvement on joint pain in children with $\mathrm{JH}$.

Limitations of this study are different and may include: (i) patients were originally selected according to old criteria and their attribution to the novel diagnostic categories (hEDS and HSD) was carried out in retrospect with possibility of wrong subclassification in selected cases; (ii) the relatively limited number of patients may have limited our ability to appreciate statistically significant associations by multivariate logistic regression analysis; (iii) what was inferred by the use of psychometric tests was not supported by more objective investigations. Accordingly, this is an exploratory study aimed at raising attention of the neuropsychological attributes of $\mathrm{JH}$, which may exist also beyond the developmental age and contribute to disability in adults. 


\section{1}

2

3

4

5

6

7

8

9

In conclusion, our work validates the FDQ-9 test as a reliable screening tool for DCD in Italian adults. The patterns of replies to the 9 items of the FDQ-9 in 230 Italian controls can be used as normative values for future studies which will apply FDQ-9 in different Italian patients' cohorts. We also demonstrated a high rate of a "likely" diagnosis of DCD in adults with hEDS/HSD. In these patients, positive FDQ-9 screening directly associates with a possible diagnosis of ADHD and number of painful joints. This work contributes to the understanding of the complex natural history of JH and the related hEDS/HSD, and open new scenarios for exploring the body-mind connections in these conditions.

\section{Acknowledgments}

The authors wish to thank the patients and parents for their kind availability and support in this study. All authors have no conflict of interest to declare. 


\section{References}

Adib, N., Davies, K., Grahame, R., Woo, P., Murray, K.J. (2005). Joint hypermobility syndrome in childhood. A not so benign multisystem disorder? Rheumatology (Oxford), 44: 744-750.

American Psychiatric Association (APA). (2000). Diagnostic and statistical manual of mental disorders, 4th Edition: DSM-IV-TR. Washington, DC: American Psychiatric Publishing.

American Psychiatric Association (APA). (2013). Diagnostic and statistical manual of mental disorders 5th edition. Arlington, VA: American Psychiatric Publishing.

Apolone, G., Mosconi, P. (1998). The Italian SF-36 Health Survey: translation, validation and norming. J Clin Epidemiol, 51, 1025-1036.

Baeza-Velasco, C., Sinibaldi, L., Castori, M. (2018). Attention-deficit/hyperactivity disorder, joint hypermobility-related disorders and pain: expanding body-mind connections to the developmental age. Atten Defic Hyperact Disord, in press.

Beighton, P., De Paepe, A., Steinmann, B., Tsipouras, P., Wenstrup, R.J. (1998). Ehlers-Danlos syndromes: revised nosology, Villefranche, 1997. Ehlers-Danlos National Foundation (USA) and Ehlers-Danlos Support Group (UK). Am J Med Genet, 77, 31-37.

Bulbena, A., Baeza-Velasco, C., Bulbena-Cabré, A., Pailhez, G., Critchley, H., Chopra, P., ... Porges, S. (2017). Psychiatric and psychological aspects in the Ehlers-Danlos syndromes. Am J Med Genet $C$ Semin Med Genet, 175, 237-245.

Bulbena, A., Pailhez, G., Bulbena-Cabré, A., Mallorquí-Bagué, N., Baeza-Velasco, C. (2015). Joint hypermobility, anxiety and psychosomatics: two and a half decades of progress toward a new phenotype. Adv Psychosom Med, 34, 143-157.

Caraceni, A., Mendoza, T.R., Mencaglia, E., Baratella, C., Edwards, K., Forjaz, M.J., ... Cleeland, C.S. (1996). A validation study of an Italian version of the Brief Pain Inventory (Breve Questionario per la Valutazione del Dolore). Pain, 65, 87-92.

Castori, M., Camerota, F., Celletti, C., Grammatico, P., Padua, L. (2010). Quality of life in the classic and hypermobility types of Ehlers-Danlos syndrome. Ann Neurol, 67, 145-146.

Castori, M., Colombi, M. (2015). Generalized joint hypermobility, joint hypermobility syndrome and Ehlers-Danlos syndrome, hypermobility type. Am J Med Genet C Semin Med Genet, 169C, 1-5.

Castori, M., Dordoni, C., Valiante, M., Sperduti, I., Ritelli, M., Morlino, S., ... Colombi, M. (2014). Nosology and inheritance pattern(s) of joint hypermobility syndrome and Ehlers-Danlos syndrome, hypermobility type: a study of intrafamilial and interfamilial variability in 23 Italian pedigrees. Am J Med Genet, 164A, 3010-3020.

Castori, M., Hakim, A. (2017). Contemporary approach to joint hypermobility and related disorders. Curr Opin Pediatr, 29, 640-649. 
Castori, M., Morlino, S., Celletti, C., Celli, M., Morrone, A., Colombi, M., ... Grammatico, P. (2012). Management of pain and fatigue in the joint hypermobility syndrome (a.k.a. Ehlers-Danlos syndrome, hypermobility type): principles and proposal for a multidisciplinary approach. Am J Med Genet A, 158A, 2055-2070.

Castori, M., Morlino, S., Celletti, C., Ghibellini, G., Bruschini, M., Grammatico, P., ... Camerota, F. (2013). Re-writing the natural history of pain and related symptoms in the joint hypermobility syndrome/Ehlers-Danlos syndrome, hypermobility type. Am J Med Genet A, 161A, 2989-3004.

Castori, M., Tinkle, B., Levy, H., Grahame, R., Malfait, F., Hakim, A. (2017). A framework for the classification of joint hypermobility and related conditions. Am J Med Genet C Semin Med Genet, $175,148-157$.

Cederlöf, M., Larsson, H., Lichtenstein, P., Almqvist, C., Serlachius, E., Ludvigsson, J.F. (2016). Nationwide population-based cohort study of psychiatric disorders in individuals with Ehlers-Danlos syndrome or hypermobility syndrome and their siblings. BMC Psychiatry, 16, 207.

Celletti, C., Castori, M., La Torre, G., Camerota, F. (2013). Evaluation of kinesiophobia and its correlations with pain and fatigue in joint hypermobility syndrome/Ehlers-Danlos syndrome hypermobility type. Biomed Res Int, 2013, 580460.

Celletti, C., Mari, G., Ghibellini, G., Celli, M., Castori, M., Camerota, F. (2015). Phenotypic variability in developmental coordination disorder: Clustering of generalized joint hypermobility with attention deficit/hyperactivity disorder, atipical swallowing and narrative difficulties. Am J Med Genet C Semin Med Genet, 169C, 117-22.

Clark, C.J., Clark, S., Dorey, C., Williams, J. (2016). Correlation of the functional difficulties questionnaire (FDQ-9) with dynamic balance using the SMART instrumented wobbleboard. Phys Ther Sport , 21, 68-74.

Clark, C., Khattab, A., Carr, E. (2014). Chronic widespread pain and neurophysicological symptoms in joint hypermobility syndrome (JHS). Int J Ther Rehabil, 21, 60-67.

Clark, C.J., Thomas, S., Khattab, A.D., Carr, E.C. (2013). Development and psychometric properties of a screening tool for assessing developmental coordination disorder in adults. Int J Phys Med Rehabil, $1,145$.

Cleeland, C.S., Ryan, K.M. (1994). Pain assessment: global use of the Brief Pain Inventory. Ann Acad Med Singapore, 23, 129-138.

De Wandele, I., Rombaut, L., De Backer, T., Peersman, W., Da Silva, H., De Mits, S., ... Malfait, F. (2016). Orthostatic intolerance and fatigue in the hypermobility type of Ehlers-Danlos Syndrome. Rheumatology (Oxford), 55, 1412-1420.

Ghibellini, G., Brancati, F., Castori, M. (2015). Neurodevelopmental attributes of joint hypermobility syndrome/Ehlers-Danlos syndrome, hypermobility type: Update and perspectives. Am J Med Genet $C$ Semin Med Genet, 169C, 107-116. 
Grahame, R., Bird, H.A., Child, A. (2000). The revised (Brighton 1998) criteria for the diagnosis of benign joint hypermobility syndrome (BJHS). J Rheumatol, 27, 1777-1779.

Jelsma, L.D., Geuze, R.H., Klerks, M.H., Niemeijer, A.S., Smits-Engelsman, B.C. (2013). The relationship between joint mobility and motor performance in children with and without the diagnosis of developmental coordination disorder. BMC Pediatr, 13, 35.

Jereczek-Fossa, B.A., Santoro, L., Alterio, D., Franchi, B., Fiore, M.R., Fossati, P., ... Orecchia, R. (2007). Fatigue during head-and-neck radiotherapy: prospective study on 117 consecutive patients. Int $J$ Radiat Oncol Biol Phys, 68, 403-415.

Kessler, R.C., Adler, L., Ames, M., Demler, O., Faraone, S., Hiripi, E.,... Walters, E.E. (2005). The World Health Organization Adult ADHD Self-Report Scale (ASRS): a short screening scale for use in the general population. Psychol Med, 35, 245-256.

Kessler, R.C., Adler, L.A., Gruber, M.J., Sarawate, C.A., Spencer, T., Van Brunt, D.L. (2007). Validity of the World Health Organization Adult ADHD Self-Report Scale (ASRS) screener in a representative sample of health plan members. Int J Methods Psychiatr Res, 16, 52-65.

Kirby, A., Davies, R., Bryant, A. (2005). Hypermobility syndrome and developmental coordination disorder: similarities and features. Int J Ther Rehabil, 12, 431-436.

Kirby, A., Davies, R. (2007). Developmental Coordination Disorder and Joint Hypermobility Syndrome-overlapping disorders? Implications for research and clinical practice. Child Care Health Dev, 33, 513519.

Koldas Dogan, S., Taner, Y., Evcik, D. (2011). Benign Joint Hypermobility Syndrome in Patients with Attention Deficit/Hyperactivity Disorders. Turk J Rheumatol, 26, 187-192.

Lisi, C., Monteleone, S., TInelli, C., Rinaldi, B., Di Natali., G., Savasta, S. (2017). Postural analysis in a pediatric cohort of patients with Ehlers-Danlos syndrome: a pilot study. Minerva Pediatr, doi: 10.23736/S0026-4946.17.04681-3.

Loeys, B.L., Dietz, H.C., Braverman, A.C., Callewaert, B.L., De Backer, J., Devereux, R.B., ... De Paepe, A.M. (2010). The revised Ghent nosology for the Marfan syndrome. J Med Genet, 47, 476-485.

Malfait, F., Francomano, C., Byers, P., Belmont, J., Berglund, B., Black, J., ... Tinkle B. The 2017 international classification of the Ehlers-Danlos syndromes. Am J Med Genet C Semin Med Genet, $175,8-26$.

Pacey, V., Tofts, L., Adams, R.D., Munns, C.F., Nicholson, L.L. (2015). Quality of life prediction in children with joint hypermobility syndrome. J Paediatr Child Health, 51, 689-695.

Piedimonte, C., Penge, R., Morlino, S., Sperduti, I., Terzani, A., ... Castori M. (2018). Exploring relationships between joint hypermobility and neurodevelopment in children (4-13 years) with hereditary connective tissue disorders and developmental coordination disorder. Am J Med Genet B Neuropsychiatr Genet, in press. 
Pierangeli, G., Turrini, A., Giannini, G., Del Sorbo, F., Calandra-Buonaura, G., Guaraldi, P., ... Cortelli, P. (2015). Translation and linguistic validation of the Composite Autonomic Symptom Score COMPASS 31. Neurol Sci, 36, 1897-1902.

Rombaut, L., Malfait, F., Cools, A., De Paepe, A., Calders, P. (2010). Musculoskeletal complaints, physical activity and health-related quality of life among patients with the Ehlers-Danlos syndrome hypermobility type. Disabil Rehabil, 32, 1339-1345.

Rombaut, L., Malfait, F., De Wandele, I., Thijs, Y., Palmans, T., De Paepe, A., Calders, P. (2011). Balance, gait, falls, and fear of falling in women with the hypermobility type of Ehlers-Danlos syndrome. Arthritis Care Res (Hoboken), 63, 1432-1439.

Scheper, M., Rombaut, L., de Vries, J., De Wandele, I., van der Esch, M., Visser, B., ... Engelbert, R. (2017). The association between muscle strength and activity limitations in patients with the hypermobility type of Ehlers-Danlos syndrome: the impact of proprioception. Disabil Rehabil, 39, 1391-1397.

Sinibaldi, L., Ursini, G., Castori, M. (2015). Psychopathological manifestations of joint hypermobility and joint hypermobility syndrome/ Ehlers-Danlos syndrome, hypermobility type: The link between connective tissue and psychological distress revised. Am J Med Genet C Semin Med Genet, 169C, 97106.

Sletten, D.M., Suarez, G.A., Low, P.A., Mandrekar, J., Singer, W. (2012). COMPASS 31: a refined and abbreviated Composite Autonomic Symptom Score. Mayo Clin Proc, 87, 1196-1201.

Smets, E.M., Garssen, B., Bonke, B., De Haes, J.C. (1995). The Multidimensional Fatigue Inventory (MFI) psychometric qualities of an instrument to assess fatigue. J Psychosom Res, 39, 315-325.

Tinkle, B., Castori, M., Berglund, B., Cohen, H., Grahame, R., Kazkaz, H., Levy, H. (2017). Hypermobile Ehlers-Danlos syndrome (a.k.a. Ehlers-Danlos syndrome Type III and Ehlers-Danlos syndrome hypermobility type): Clinical description and natural history. Am J Med Genet C Semin Med Genet, $175,48-69$.

Tinkle, B.T., Bird, H.A., Grahame, R., Lavallee, M., Levy, H.P., Sillence, D. (2009). The lack of clinical distinction between the hypermobility type of Ehlers-Danlos syndrome and the joint hypermobility syndrome (a.k.a. hypermobility syndrome). Am J Med Genet A, 149A, 2368-2370.

Ustun, B., Adler, L.A., Rudin, C., Faraone, S.V., Spencer, T.J., Berglund, P., ... Kessler, R.C. (2017). The World Health Organization Adult Attention-Deficit/Hyperactivity Disorder Self-Report Screening Scale for DSM-5. JAMA Psychiatry, 74, 520-526. 


\section{Legends to Figures}

Figure 1

Categorical diagnoses of the 105 enrolled patients. Comparison between the "old" nomenclature (Villefranche and Brighton criteria) and the 2017 International Classification of Ehlers-Danlos syndromes and related disorders. Sixty-nine patients met the Villefranche criteria (+/- Brighton criteria) and 36 the Brighton criteria only. Fifty-eight of those meeting the Villefranche criteria also met the 2017 criteria of hypermobile Ehlers-Danlos syndrome. The remaining eleven patients meeting the Villefranche criteria and all individuals respecting the Brighton criteria only were reclassified as hypermobility spectrum disorders.

Figure 2

Distribution of the Beighton score among the 105 patients with hypermobile Ehlers-Danlos syndrome/hypermobility spectrum disorders.

Figure 3

Patterns of replies to the nine items of the FDQ-9 in the 230 Italian controls.

Figure 4

Patterns of replies to the nine items of the FDQ-9 in the hypermobile Ehlers-Danlos syndrome/hypermobility spectrum disorder patients' cohort and in the controls. Both groups are composed of 105 individuals (see text for more details). Significance of the differences is reported as p-values. hEDS/HSD = hypermobile Ehlers-Danlos syndrome/hypermobility spectrum disorders.

\section{Figure 5}

Pattern of replies to the FDQ-9 questionnaire in the patients' and controls' groups. Replies are classified in $\leq 18=$ no functional difficulties; $19-21=$ at risk of having functional difficulties; $>21=$ with one or more functional difficulties. hEDS/HSD = hypermobile Ehlers-Danlos syndrome/hypermobility spectrum disorder.

Figure 6 
Relationship between the outcomes (positive vs negative = "at risk" vs "not at risk") to the FDQ-9 and ASRS-V1.1 in the patients' sample.

John Wiley \& Sons, Inc. 


\section{Tables}

Table I. Original and Italian versions of the FDQ-9 questionnaire.

\begin{tabular}{|c|c|c|}
\hline $\begin{array}{l}\text { Item } \\
\text { no. }\end{array}$ & FDQ-9 (Original Version) & FDQ-9 (Italian Version) \\
\hline 1 & As a child, how good was your hand writing? & Da bambino com'era la tua scrittura (n.d.a. scrittura a mano su carta)? \\
\hline 2 & $\begin{array}{l}\text { As a child, were you good at team games that involved balls? i.e. football, } \\
\text { netball, basketball }\end{array}$ & Da bambino come te la cavavi con gli sport di squadra con la palla? \\
\hline 3 & As a child, how did others rate your coordination? & $\begin{array}{l}\text { Da bambino gli altri (genitori, insegnanti, amici) come consideravano la tua } \\
\text { coordinazione? }\end{array}$ \\
\hline 4 & $\begin{array}{l}\text { As an adult, how good are you at avoiding obstacles, like bumping into } \\
\text { doors? }\end{array}$ & $\begin{array}{l}\text { Da adulto quanto riesci ad evitare gli ostacoli (p.e. attraversare una porta } \\
\text { senza sbattere contro lo stipite/maniglia, evitare di urtare contro gli angoli } \\
\text { di un tavolo)? }\end{array}$ \\
\hline 5 & $\begin{array}{l}\text { As an adult, how good are you at organizing yourself? i.e. getting ready for } \\
\text { work or for a meeting }\end{array}$ & $\begin{array}{l}\text { Da adulto quanto riesci ad organizzarti (p.e. prepararti in tempo per lavoro o } \\
\text { per un incontro? }\end{array}$ \\
\hline 6 & As an adult, how good were you at catching a ball one handed? & $\begin{array}{l}\text { Da adulto quanto sei bravo a prendere al volo la palla (oppure, oggetti di } \\
\text { piccole dimensioni, ecc.)? }\end{array}$ \\
\hline 7 & $\begin{array}{l}\text { As an adult, how good are you at balancing on a bike, in a bus or train, or on } \\
\text { skis? }\end{array}$ & $\begin{array}{l}\text { Da adulto quanto sei capace a mantenere l'equilibrio in bici/sull'autobus/sul } \\
\text { treno/in barca? }\end{array}$ \\
\hline 8 & $\begin{array}{l}\text { As an adult, how good are you at using your hands i.e. to do jobs around the } \\
\text { home, DIY, sewing or using scissors? }\end{array}$ & $\begin{array}{l}\text { Da adulto quanto sei bravo ad usare le mani, p.e. nel bricolage, nel taglio e } \\
\text { cucito? }\end{array}$ \\
\hline 9 & As an adult, how good is your hand writing now? & Da adulto com'é la tua scrittura (n.d.a. scrittura a mano su carta)? \\
\hline
\end{tabular}


Table II. Results of the univariate analysis comparing FDQ-9 with BPI, MFI-20, COMPASS-31 and SF36 in 105 adults with hEDS/HSD.

\begin{tabular}{|c|c|c|c|c|c|}
\hline \multirow[t]{2}{*}{ Items } & \multicolumn{2}{|c|}{$\begin{array}{c}\text { FDQ-9 negative ( } \leq 21 ; \\
\text { no. } 43)\end{array}$} & \multicolumn{2}{|c|}{$\begin{array}{l}\text { FDQ-9 positive (>21; } \\
\text { no. } 62 \text { ) }\end{array}$} & \multirow[t]{2}{*}{$p$-value } \\
\hline & Median & Range & Median & Range & \\
\hline \multicolumn{6}{|l|}{ Brief pain inventory } \\
\hline Bodily pain (painful points) & 5.0 & $0-21$ & 10.0 & $1-24$ & 0.002 \\
\hline Joint pain (painful points) & 2.0 & $0-12$ & 5.5 & $0-12$ & $<0.0001$ \\
\hline Intensity, maximum & 6.0 & $0-10$ & 8.0 & $3-10$ & 0.006 \\
\hline Intensity, minimum & 3.0 & $0-10$ & 4.0 & $0-9$ & 0.106 \\
\hline Intensity, mean & 4.0 & $0-10$ & 6.0 & $2-10$ & 0.002 \\
\hline Intensity, current & 4.0 & $0-10$ & 6.0 & $0-9$ & 0.013 \\
\hline Interference, GA & 5.0 & $0-10$ & 7.0 & $0-10$ & 0.012 \\
\hline Interference, mood & 5.0 & $0-10$ & 6.0 & $0-10$ & 0.038 \\
\hline Interference, rowing & 5.0 & $0-10$ & 7.0 & $0-10$ & 0.005 \\
\hline Interference, WA & 5.0 & $0-10$ & 8.0 & $0-10$ & $<0.0001$ \\
\hline Interference, SR & 3.0 & $0-10$ & 5.0 & $0-10$ & 0.003 \\
\hline Interference, sleeping & 5.0 & $0-10$ & 8.0 & $0-10$ & 0.001 \\
\hline Interference, WTL & 3.0 & $0-10$ & 6.0 & $0-10$ & 0.002 \\
\hline \multicolumn{6}{|l|}{$\begin{array}{l}\text { Multidimesional fatigue } \\
\text { inventory } 20\end{array}$} \\
\hline MFI20-GF & 13.0 & $8-20$ & 12.5 & $8-20$ & 0.496 \\
\hline MFI20-PF & 15.0 & $6-20$ & 14.0 & $8-20$ & 0.497 \\
\hline MFI20-RA & 11.0 & $4-20$ & 12.0 & $4-20$ & 0.085 \\
\hline MFI20-RM & 13.0 & $6-20$ & 14.0 & $8-20$ & 0.503 \\
\hline MFI20-MF & 13.0 & $8-19$ & 13.5 & $7-18$ & 0.038 \\
\hline \multicolumn{6}{|l|}{$\begin{array}{l}\text { Composite autonomic } \\
\text { symptom score } 31\end{array}$} \\
\hline COMPASS31-OI & 5.0 & $0-10$ & 6.5 & $0-10$ & 0.001 \\
\hline COMPASS31-VM & 2.0 & $0-6$ & 3.0 & $0-6$ & 0.016 \\
\hline COMPASS31-SM & 3.0 & $0-6$ & 4.0 & $0-6$ & 0.003 \\
\hline COMPASS31-GI & 10.0 & $0-20$ & 14.0 & $1-21$ & 0.001 \\
\hline COMPASS31-B & 1.0 & $0-6$ & 3.0 & $0-9$ & 0.003 \\
\hline COMPASS31-PM & 9.0 & $0-14$ & 10.0 & $0-15$ & 0.090 \\
\hline Short form 36 & & & & 2 & \\
\hline SF36-PA & 60.0 & $0-100$ & 45.0 & $5-100$ & 0.004 \\
\hline SF36-PRL & 25.0 & $0-100$ & 0.0 & $0-100$ & 0.003 \\
\hline SF36-PP & 41.0 & $0-100$ & 22.0 & $0-72$ & 0.092 \\
\hline SF36-GH & 25.0 & $0-76$ & 20.0 & $0-61$ & 0.147 \\
\hline SF36-V & 40.0 & $0-80$ & 22.50 & $5-85$ & 0.005 \\
\hline SF36-SA & 50.0 & $0-100$ & 37.0 & $0-100$ & 0.004 \\
\hline SF36-ERL & 66.0 & $0-100$ & 16.50 & $0-100$ & 0.006 \\
\hline SF36-MH & 60.0 & $4-100$ & 52.00 & $12-92$ & 0.012 \\
\hline SF36-PH & 107.0 & $5-300$ & 82.0 & $10-272$ & 0.002 \\
\hline SF36-GH+V & 70.0 & $5-151$ & 45.0 & $5-146$ & 0.013 \\
\hline SF36-PEH & 183.0 & $16-300$ & 112.0 & $16-292$ & 0.001 \\
\hline
\end{tabular}

Significant $p$-values are in bold. The analysis was carried out by the Mann-Whitney non-parametric test. Abbreviations for the brief pain inventory (BPI): GA, general activity; SR, social relations; WA, working activity; WTL, will to live. Abbreviations for the multidimensional fatigue inventory 20 (MFI20): MFI20-GF, general fatigue; MFI20-PF, physical fatigue; MFI20-RA, reduction of activities; MFI20- 
RM, reduction of motivations; MFI20-MF, mental fatigue. Abbreviations for the composite autonomic symptom score 31: COMPASS31-B, bladder functions; COMPASS31-GI, gastrointestinal functions; COMPASS31-OI, orthostatic intolerance; COMPASS31-PM, pupillomotor functions; COMPASS31-SM, secretomotor functions; COMPASS31-VM, vasomotor functions. Abbreviations for the short-form 36 (SF-36): SF36-PA, physical activity; SF36-PRL, physical role limitations; SF36-PP, physical pain; SF36-GH, general health; SF36-V, vitality; SF36-SA, social activity; SF36-ERL, emotional role limitations; SF36-MH, mental health; SF36-PH, physical health; SF36-GH+V, general health plus vitality; SF36-PEH, psycho-emotional health. 


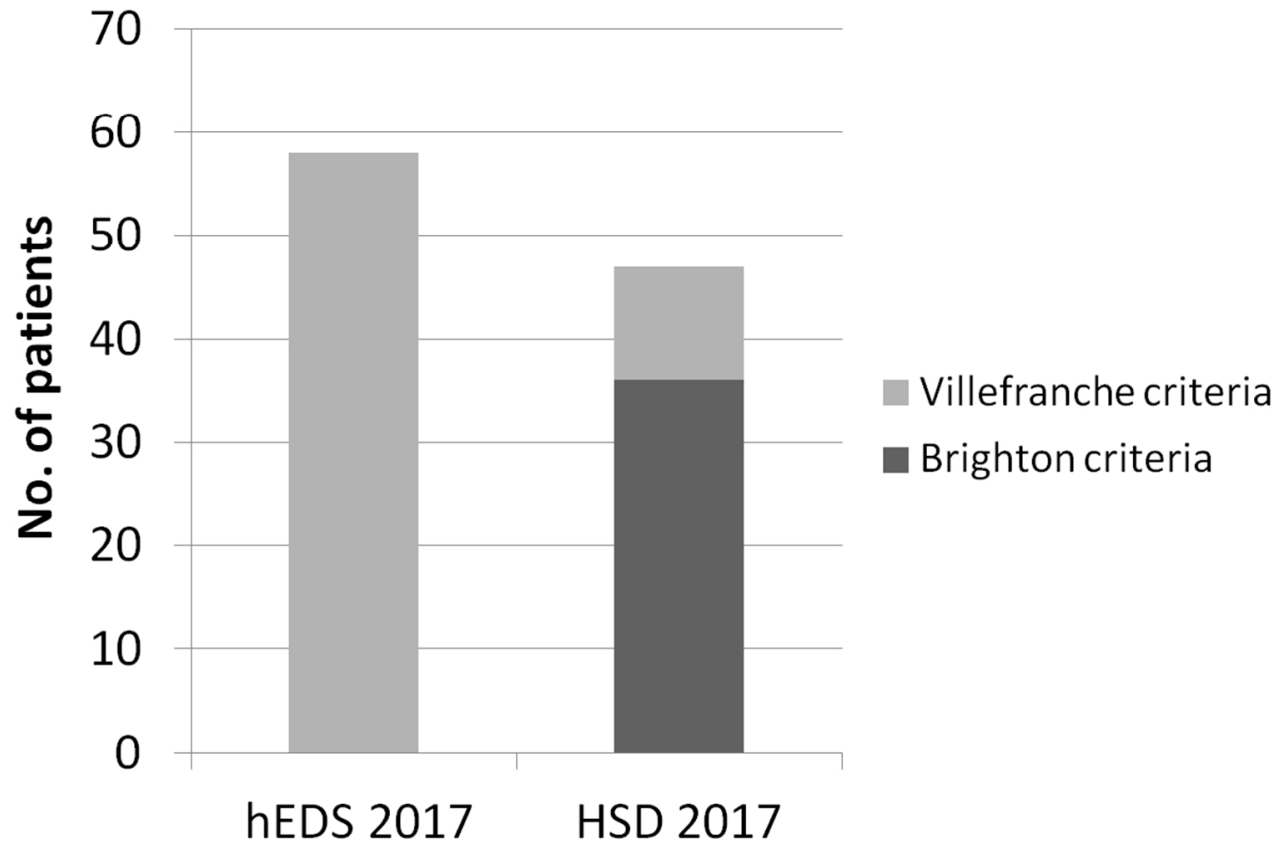

Categorical diagnoses of the 105 enrolled patients. Comparison between the "old" nomenclature (Villefranche and Brighton criteria) and the 2017 International Classification of Ehlers-Danlos syndromes and related disorders. Sixty-nine patients met the Villefranche criteria (+/- Brighton criteria) and 36 the Brighton criteria only. Fifty-eight of those meeting the Villefranche criteria also met the 2017 criteria of hypermobile Ehlers-Danlos syndrome. The remaining eleven patients meeting the Villefranche criteria and all individuals respecting the Brighton criteria only were reclassified as hypermobility spectrum disorders.

$207 \times 142 \mathrm{~mm}(150 \times 150 \mathrm{DPI})$ 


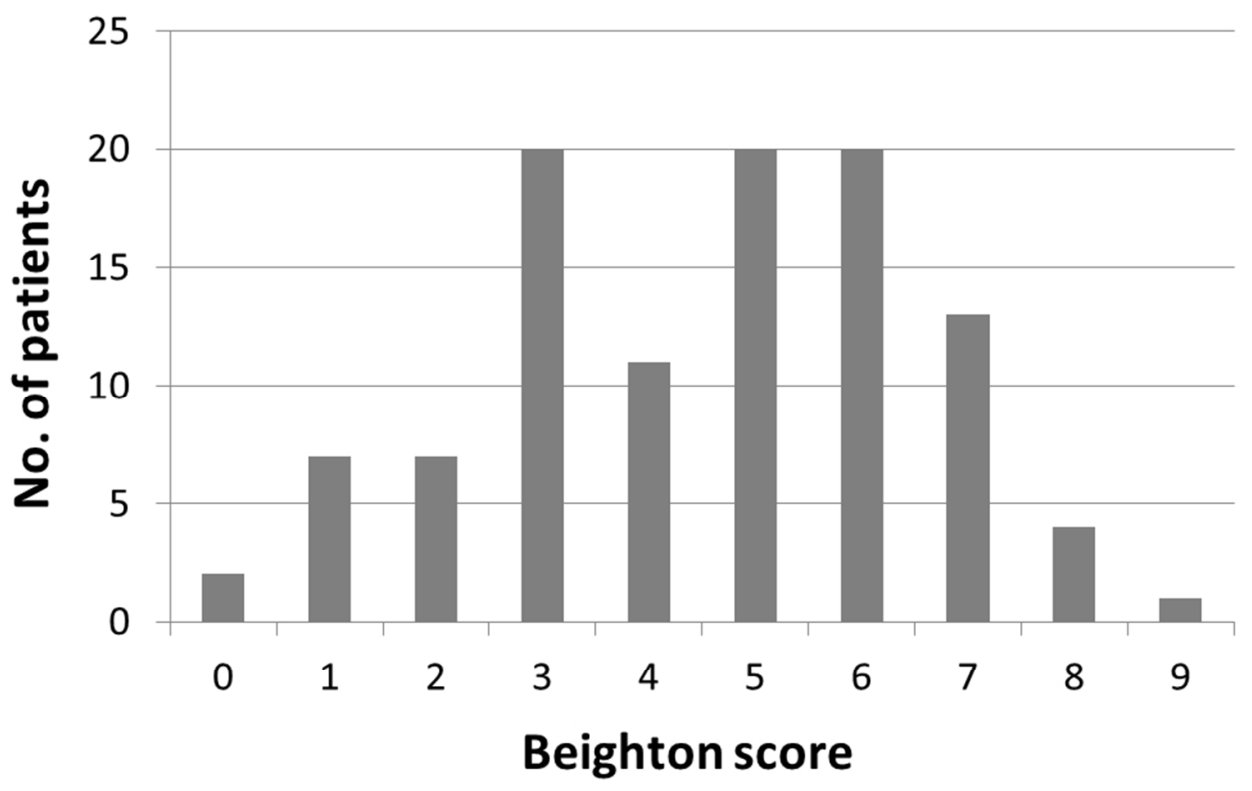

Distribution of the Beighton score among the 105 patients with hypermobile Ehlers-Danlos syndrome/hypermobility spectrum disorders.

$171 \times 108 \mathrm{~mm}(150 \times 150 \mathrm{DPI})$ 


\section{1 (very good) $\square 2$ (good) $\square 3$ (poor) $\square 4$ (very poor)}

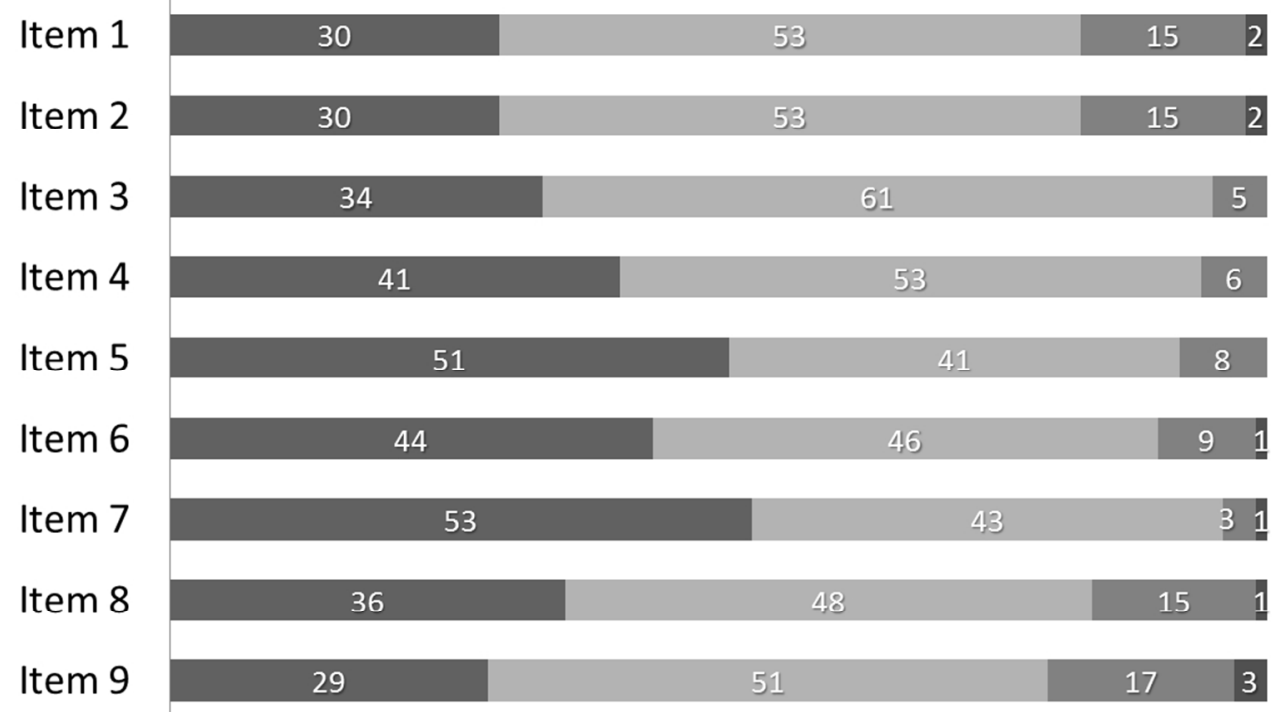

Patterns of replies to the nine items of the FDQ-9 in the 230 Italian controls.

$193 \times 125 \mathrm{~mm}(150 \times 150 \mathrm{DPI})$ 
Patterns of replies to the nine items of the FDQ-9 in the hypermobile Ehlers-Danlos syndrome/hypermobility spectrum disorder patients' cohort and in the controls. Both groups are composed of 105 individuals (see text for more details). Significance of the differences is reported as $p$-values. hEDS/HSD $=$ hypermobile Ehlers-Danlos syndrome/hypermobility spectrum disorders.

$196 \times 110 \mathrm{~mm}(150 \times 150 \mathrm{DPI})$ 


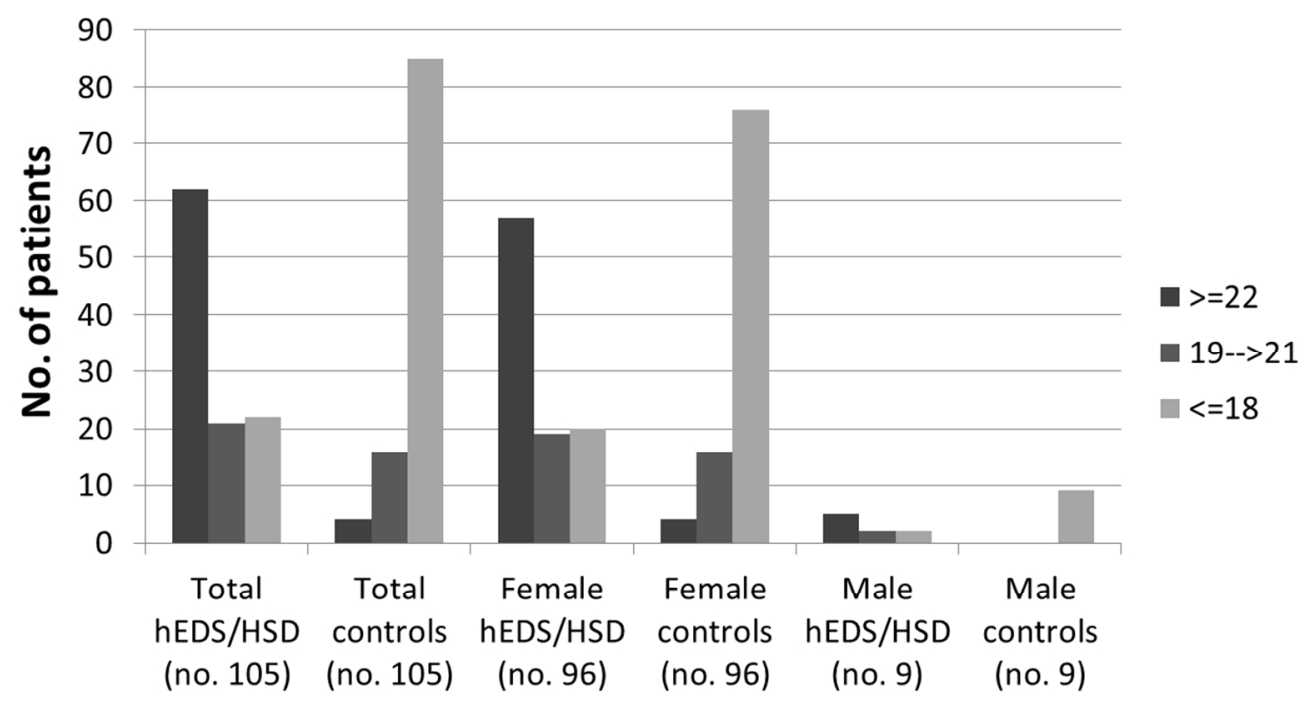

Pattern of replies to the FDQ-9 questionnaire in the patients' and controls' groups. Replies are classified in $\leq 18=$ no functional difficulties; $19-21=$ at risk of having functional difficulties; $>21=$ with one or more functional difficulties. hEDS/HSD = hypermobile Ehlers-Danlos syndrome/hypermobility spectrum disorder.

$209 \times 115 \mathrm{~mm}(150 \times 150 \mathrm{DPI})$ 


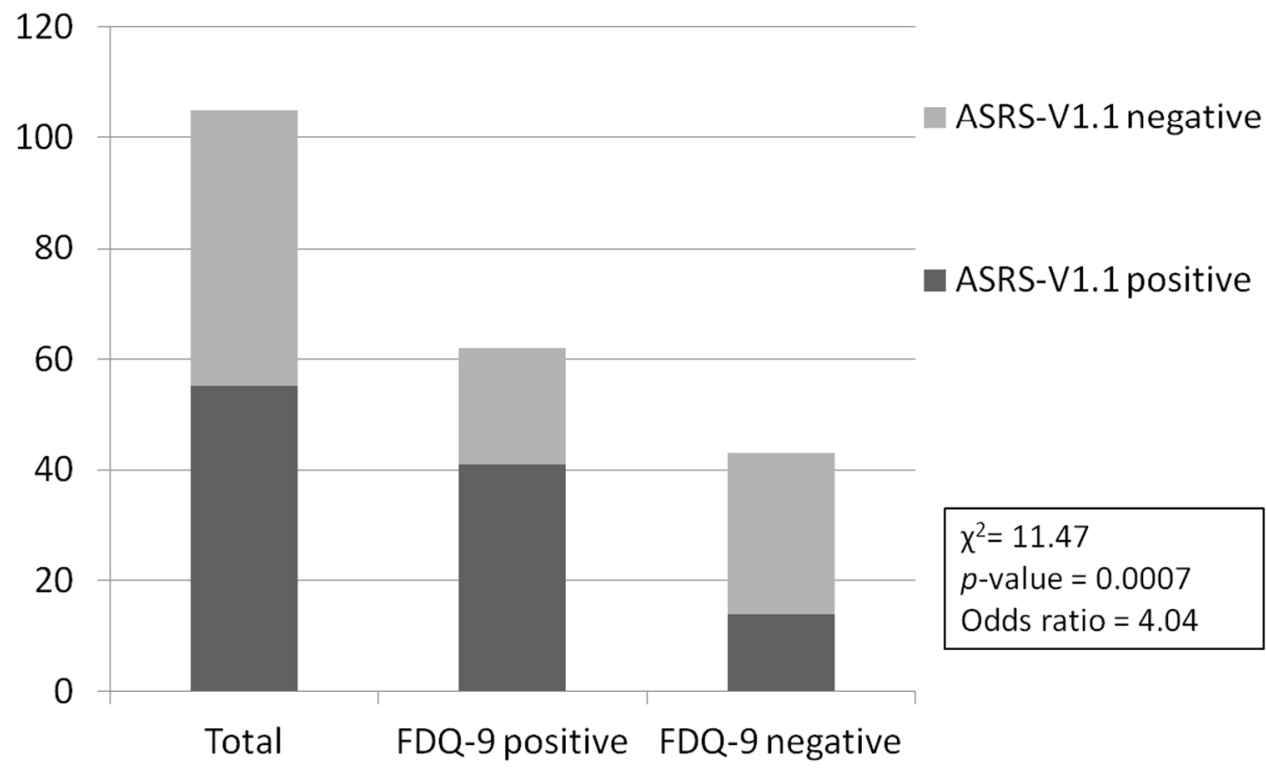

Relationship between the outcomes (positive vs negative = "at risk" vs "not at risk") to the FDQ-9 and ASRS-V1.1 in the patients' sample.

\section{$212 \times 127 \mathrm{~mm}(150 \times 150 \mathrm{DPI})$}


Fondazione

\author{
Casa Sollievo della Sofferenza \\ Opera di San Pio da Pietrelcina \\ OSPEDALE \\ Istituto di Ricovero e Cura a Carattere Scientifico \\ Viale Cappuccini - 71013 San Giovanni Rotondo (FG)
}

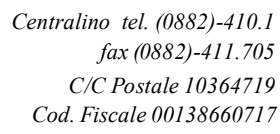

Centralino tel. (0882)-410.1 fax (0882)-411.705 C/C Postale 10364719 Cod. Fiscale 00138660717

Rome, July 24th 2018

Division of Medical Genetics

IRCCS-Casa Sollievo della Sofferenza

Poliambulatorio $-2^{\text {nd }}$ Floor

Viale Padre Pio snc

71013 San Giovanni Rotondo

Foggia, Italy

Phone: +39-0882-416288

Fax: +39-0882-411616

Email: m.castori@operapadrepio.it

\title{
Ming T. Tsuang \\ University of California, San Diego \\ Harvard University \\ Senior Editor, American Journal of Medical Genetics Part B Neuropsychiatric Genetics \\ Stephen V. Faraone \\ SUNY Upstate Medical University \\ Editor, American Journal of Medical Genetics Part B Neuropsychiatric Genetics
}

RE: replies to the intermediate revision for "Italian validation of the functional difficulties questionnaire (FDQ-9) and its correlation with major determinants of quality of life in adults with hypermobile Ehlers-Danlos syndrome/hypermobility spectrum disorder (NPG-18-0060)"

\section{Dear prof. Tsuang and prof. Faraone,}

I would be grateful to you if you will consider the revised version of the above mentioned paper for publication in the American Journal of Medical Genetics Part B Neuropsychiatric Genetics as a "Research Article".

Here you can find our replies to the reviewers' comments.

\section{REVIEWER \#1}

General comment. This is a well constructed study, the data appropriately analyzed, the literature appropriately considered, and the subject important to this area of Medicine.

Reply. All authors thank this reviewer for her/his general consideration of the manuscript.

Grammar/typing corrections. My comments are about typographical errors and a couple of sentences that seem incomplete. Presumably these will get picked up by the editorial team and lead author anyway but here is what I found $(P=$ page,$L=$ closest number on left though these do not match up well to the actual text lines):

1. At several places there the misspelling "test/ritest" (P2 L20; P5 L41; P10 L25)

2. I suggest INdistinguishable (modern English) rather than UNdistinguishable (P3, L30)

3. At several places the second in list is numbered 'i' not 'ii' (P2 L14; P4 L26)

4. P4 L53 to P5 L3 the sentence is not complete.

5. P5 L14 the word 'according' should be 'accordance' John Wiley \& Sons, Inc. 
6. P5 L7 the word 'Ethic' should be 'Ethics' I think

7. P6 L26-27 - the sentence I believe would read better as "The cut-off for suspecting ADHD in adults is currently 4 or more positive answers"

8. P7 L9 - the word 'by' change to 'of' i.e. "...composed of four..."

9. P7 L22 - the word 'has' to 'have' i.e. "...areas have..."

10. $P 9 L 13$ - the italics should read RDQ-9

11. P9 L30 - 'was' should be 'is' i.e. "...controls is summarized..."

12. P9 L41 and P10 L4 - 'were' should be 'are' i.e. "The results are summarized in Fig. 5." and "...are reported in..."

13. P9 L52 - 'at' should be 'for i.e. "... resulted positive for the..."

14. P10 Senctence $L 7$ to 12. I suggest this should read at the beginning as "The strongest associations (p-value <0.002) were:...", and that the comment about work should read "...pain interference with work and sleep,..."

15. P10 L25 delete the 's' from the word controls

16. P 10 L33 change 'of' to 'from' or 'with' i.e. "...to suffer from clinic..."

17. P 11 L46 change the word 'suspect' to 'suspicion'

18. $P 12$ L9 $i$ think the words 'has' and 'been' are missing i.e. "...confirms what has previously been..."

19. P12 L43-46 point (ii) - this needs rewording - perhaps "...may have limited our ability to appreciate statistically significant associations by mulivariate..."

Reply. All corrections were included in the present version of the paper.

\section{REVIEWER \#2}

General comment. The present article emphasizes the impact of developmental coordination disorders and impaired proprioception on adults' quality of life; [...]

Reply. All authors thank this reviewer for her/his general consideration of the manuscript.

Comment. [...] on this subject I would recommend citing the article by Lisi $C$ et al. concerning impaired postural stability in EDS pediatric patients. In the paper a cross-sectional study matching a group of 12 cEDS and hEDS patients and a group of 12 healthy controls was performed, and was analized the displacement of the centre of pressure both with open and closed eyes using four parameters (standard deviation of antero-posterior excursion, standard deviation of latero-lateral excursion, sway path and sway area). The results showed significant impairment of postural control (specially of sway area) in patients' group.

I would suggest to cite the article approximately at page 12, lines 24-30.

Reply. The suggested reference was added in the manuscript by citing it exactly in the proposed paragraph.

\section{REVIEWER \#3}

General comment. It's an interesting manuscript.

Reply. All authors thank this reviewer for her/his general consideration of the manuscript.

Comment 1. I have a principal question: which method have you used to validate the italian version of the FDQ-9 scale?

Reply 1. The Italian version of the FDQ-9 was validated by a three-step analysis, including translation, cross-cultural adaptation and test/retest reliability analysis. In particular, the test/retest reliability analysis is a reproducibility test, at present, considered a highly confident tool for validating the translation of psychometric tests.

Comment 2. Please add a table with the demographic caractheristics of the study group compared with the control group and specify if there are significant differences.

Reply 2. Comparison among the patients' and controls' demographic characteristics (with p-values, all not significant) were reported on pages 9\&10, as follows: "Ninety-six were females and 9 males (female/male ratio: 10.6/1) with an age ranging from 18 to 62 years (mean: 36.9 years, standard deviation: 12.08). Their diagnosis was revised retrospectively, according to the 2017 International Classification of EDS and related disorders, and, hence, translated into the novel nosology of hEDS and HSD (Fig. 1). The distribution of the Beighton score in the patients' sample is reported in Fig. 2. The 230 controls from the general population included 138 females and 92 males with an age range of 19 to 95 years (mean 38.43 years; standard deviation: 12.20). Compared to the 105 patients, the sex-and age-matched 105 controls were 96 females and 9 males (p-value: 1.00) with an age range from 19 to 62 years (mean: 37.2 years, standard deviation: 12.31 ; p-value: 0.87 )".

Given the very limited number of features to compare we decided to do not add a further table. Neverthless, we will surely add it in case the reviewer will consider this crucial for the quality of the paper.

All contributors have contributed sufficiently to the intellectual content of this submission. They have also read and approved the submitted version of the manuscript.

The authors declare that this work has not been published and/or submitted elsewhere, and that no conflict of interest exists concerning this manuscript.

John Wiley \& Sons, Inc.

This is the first submission of this paper. 
Yours faithfully,

Marco Castori, MD, PhD

Chief of the Division

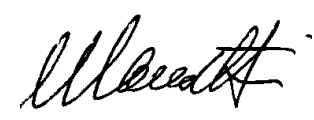

7

8

9

10

11

12

13

14

15

16

17

18

19

20

21

22

23

24

25

26

27

28

29

30

31

32

33

34

35

36

37

38

39

40

41

42

43

44

45

46

47

48

49

50

51

52

53

54

55

56

57

58

59

60

John Wiley \& Sons, Inc. 